\title{
Editorial
}

William P.S. McKay MD, *

Peter H. Gregson PhD, $\dagger$

Benjamin W.S. McKay PhD
Transfer-function analysis in anesthesia research
E congratulate Fujiwara et al. for introducing to the anesthesiology community the technique of transfer-function analysis (TFA), also known as frequency response analysis, in this issue of the Journal. ${ }^{1}$ Transferfunction analysis enables us to deduce information about the behaviour of a system by comparing output signals with input signals. ${ }^{2}$ It yields more information about the physiological processes hidden deep within than does the simple study of output signals. It is noninvasive, although it can make use of invasive monitor signals. Transfer-function analysis can give unique insights into the many situations in anesthetic practice where drugs alter the complex interactions of periodic physiological functions of interest to anesthesiologists (circulation, respiration, many neurological regulators).

Although it is relatively new to medical research, ${ }^{3}$ TFA is by no means "experimental". It has been well established in engineering for many years. Its subtleties, shortcomings, and controversies have long been well worked out. Its mathematical underpinnings are even older (Euler, 1707 - 1783; Fourier, 1786 - 1830 among others).

\section{The signal}

A signal is a means to convey information. ${ }^{4}$ We will confine this discussion to the many physiological processes which can be studied by transducing a physical signal (sound, pressure, motion, etc) into changes in voltage (a $y$-axis dependent variable) over time (the $x$-axis independent variable) which can then be conveyed into a computer by digitisation. Then, where we suspect that physiological stimuli (input) cause or influence physiological responses (output), then diagrammatically, we have:

Physiological signals (input and output) $\rightarrow$ transducer $\rightarrow$ changing voltage $\rightarrow$ analogue to digital device rapidly and regularly sampling the voltage $\rightarrow$ two computer files of a set of numbers (input and output).
Each number resulting from digitisation is a sample of the voltage at its sampling time. Thus, each holds information about voltage in its magnitude, and about time in its position in the list of numbers. Such a list of numbers is called a vector.

The changing physiological signals must be simultaneously transduced and digitised. Transfer-function analysis compares the input and output vectors. Both transduction and digitisation introduce sampling errors in both amplitude and time. For good instruments, the errors are small, normally-distributed, and random. Poor instruments give large and/or systematically biased errors.

The one-dimensional plot of $\mathrm{y}$-axis voltage $p s \mathrm{x}$-axis time is said to show the signal in the time-domain. Such signals are of two main categories: deterministic (for which there is a mathematical relationship that gives a specific value for voltage at every time), and random or stochastic (for which the value at any given time can be described only by a statistical probability). Deterministic signals are further classified, briefly, as either periodic (they have a regularly repeating pattern), or transient (they do not). Periodic signals can be described mathematically by periodic functions formed by summing sines and cosines with appropriate frequencies and amplitudes. Transfer-function analysis is used most readily to study the effects of a system on periodic signals. A related technique, convolution/deconvolution, is best used to study a system's effects on transients. Remarks about the usefulness of TFA in anesthesia research also apply to convolution/deconvolution.

Transfer-function analysis theory assumes that the signals are in a steady state. That is, periodic signals produce a constantly repeating wave-form, or stochastically changing signals have constant statistical probabilities. Thus, an experiment to study drug effect would have input and output vectors compared by TFA before giving drug, and again after a steady physiologi- 
cal state has been reached with drug aboard. Then TFAs would be compared to deduce drug effect.

Transfer-function analysis is much simplified if the input is a known white signal. The Fujiwara paper makes use of a known stochastically-generated white respiratory train to take advantage of this. White light contains equal energy at all frequencies. Stemming from this, signals with equal energy at all frequencies are called white. Theoretical white signals contain all frequencies from zero to infinity. If energy occurs only within a range or band of frequencies, the sound is said to be band-limited within this range.

\section{Phase}

Many anesthesiologists are accustomed to interpreting electroencephalogram amplitude power spectra from Fourier transforms where phase information is discarded. Phase spectra are less intuitive. Periodic signals have a recurring cycle of voltage amplitude at a regularly recurring time interval $(=1 /$ frequency); thus they are, at any given moment, in a specific phase of their cycle. They are thus amenable to the study of both phase and amplitude, and of the relationships of one to the other. These relationships, difficult to discern in a timedomain plot, are best seen by plotting both amplitude and phase versus frequency in a frequency-domain plot. The transfer-function plot is a special frequency-domain plot which shows the shifting of phase and scaling of amplitude of input to output for each frequency.

The transformations of an input signal by a system to produce the output may take time. This delay has the effect of shifting the phase of component sinusoids in the output: a frequency-dependent phase-shift. Thus, an input signal would always be expected to lead the output signal in the transfer function phase plot. Sometimes, the output phase appears to lead the input. There are three related ways this can happen: 1 ) input leads output in time by more than $180^{\circ} ; 2$ ) input and output are inversely related (signal inversion) with little actual delay; and 3) measurement error. Simple examples will make this clear:

1) LONG PHASE LAGS. If input leads output by more than $180^{\circ}$, a spurious lag is the result of TFA convention. On a clock face, let the hands go $180^{\circ}$ from 12 to 6 and minus $180^{\circ}$ from 12 to 6 the other way. If a triggering event happens at 12 o'clock (zero degrees input) that causes a response nine hours later, at 9 o'clock (-90 degrees output), the triggering event actually precedes the response by nine hours. However, the TFA convention is: output cycles that are negatively phase shifted are said to lead the input. Transfer-function analysis would measure the phase delay $120^{\circ}$ 'clock to $90^{\prime}$ 'clock as minus $90^{\circ}$ and insist that the output cycle leads the input by three hours. This is an artifact of long phase lags in steady-state periodic systems.

Fill a long balloon with water. Perform gentle twofinger neonatal cardiac massage at various frequencies and amplitudes at one end with one hand (periodic input), while delicately sensing the wave motion (periodic output) transmitted to the other end with the other hand. There is an evident delay of the input wave reaching the other end, and, at some frequencies and amplitudes, the input wave is "down" while the output is "up" due to the true time delay inherent in the system. Output may appear to lead input at some frequencies and amplitudes.

2) Signal inversion. Next, picture a teeter-totter driven at one end (input) to rock in a sinusoidal fashion. Vertical motion is transduced at each end. The output signal is $\mathbf{1 8 0}$ out of phase with input, but because the system produces instantancous total signal inversion, there is no actual time lag. Transducing the vertical motion of marks on a system of two meshed gears (gear 1: input: mark at 12 o'clock; gear2: output: mark at 9 o'clock) can create instantaneous partial signal inversion such that output appears to lead input.

3) MeAsurement error. For frequencies with a very small transfer of energy (small $|\mathrm{H}(\mathrm{q})|$, the amplitude in the TFA), the phase information exaggerates sampling error by a kind of mathematical parallax. A small $|\mathrm{H}(\mathrm{q})|$ value produces meaningless phase information, which is why both magnitude and phase must always be considered together.

Finally, to complicate matters, combinations of all three mechanisms occur. Thus, it is helpful to know as much as possible about the workings of the system to interpret phase shifts correctly.

\section{The system}

A system in mathematics changes one or more inputs to one or more outputs. Many physiological processes, in their mid-ranges, which is where organisms "live" most of their lives, show a linear response to changing influences; that is, the response is proportional to the influence. This enables useful mathematical modeling by linear methods such as TFA and convolution/deconvolution. Linear systems demonstrate the properties of scaling (multiplying the voltage by a scaling factor is valid) and superposition or additivity (response to the sum of two input signals is the sum of the responses to each).

\section{The process}

Briefly, the output signal is compared to the input signal by first using a Fast Fourier Transform, a complex- 
number procedure, to transform both input and output vectors to frequency-domain functions which retain their amplitude and phase information. The function which transforms input to output (the system function) is then estimated by dividing the complex-valued output function by the complex-valued input function.

The statistical validity of the process is then tested by a complex-number version of linear regression called a coherence function, which yields a value from zero to one. A low coherence is found where 1) an input or output signal is contaminated with noise, or 2) the output signal is causally unrelated to the input signal, or 3 ) the system is non-linear. A high coherence means a high probability that the output signal is causally related to the input signal and that the system is linear.

Plots are then examined and deductions made about system behaviour. The more that is known about the structure and functioning of the physiological system, the more it is possible to correctly interpret the TFA.

\section{Role in anesthesia research}

Much of our knowledge of physiology and pharmacology derives from experiments on young healthy quadrupeds, anesthetised with barbiturate, upside down in a most unnatural (for them) supine position. Our patients, on the other hand, are old, ill, right side up bipeds. Historically, we have extrapolated animal findings to patients reasonably successfully. But perhaps we could do better. Transfer-function analysis is not invasive. An experimental model, much like Fujiwara's, could be used in human studies of many drugs that have effects on the circulation. It might well yield new insights into their physiological effects, especially in special populations (newborns, elderly, patients with diseases such as atherosclerosis) which may behave differently from young adult laboratory animals.

A population of special interest for this technique is patients with atrial fibrillation, which provides a naturally occurring, known, stochastically-generated (random) heart-rate-variability signal. Likewise, a stochastic input signal, rather than the regular, periodic one used universally at present, would be easy to program for visual, auditory, and somatosensory evoked potentials. Transfer function analysis might well clarify the muddy waters of drug interactions with evoked potentials.

\section{Caveats}

Nothing of the natural world is truly linear. Although many phenomena are close enough for linear methods to be useful, several physiological phenomena are not linear. We must always view results with caution, and test our assumptions as well as possible. Researchers are reminded that TFA is something to be tried and tested against what is already known. It is not a magic box that hands out answers, with no thought required.

We need to examine the picture of the $|\mathrm{H}(\mathrm{q})|$ function, and give physical meaning to its bumps and troughs. For example, we can see that sea-water, when sunlight (a white-light signal) shines through it, is blue/green, i.e. we easily carry out the "transfer function analysis" in our visual cortex. But to say why it is blue/green, we need to understand the behaviour of photons in that water: a difficult next step. It is common for TFA to focus nicely the next questions to be asked rather than to provide mechanistic answers.

The bumps and the troughs of $|\mathrm{H}(\mathrm{q})|$, wherever the coherence is strong, tell us that this is a frequency we bave to explain. To find an explanation, we look for physiological processes with those frequencies (or contaminating noise from other instruments in the

\section{Some terminology analogues}

Term
periodic signal
periodic signal frequency
periodic signal amplitude
periodic signal phase
transient input
transient output
deterministic signal
stochastic signal
time to frequency domain transform
frequency to time domain transform
TFA

Physiology
pulse
musical pitch
sound loudness (power)
sinus to AV node delay
bolus
lung crackle
pace-maker driven pulse
pulse train in atrial fbrillation
inner ear cochlea
motor nerve traffic driving cyclists legs
sunlight through water (see text)

Engineering

waves (any media)

"60 cycle" alternating current

AC voltage (not power)

time lag of sound and its echo

triggered seismic explosion

bridge collapse

sine-wave generator

sound of a babbling brook

FM transmitter

FM receiver

audio equipment testing

\author{
Mathematics \\ sine wave \\ sine function angular \\ rotation \\ power spectrum \\ sine function phase angle \\ delta function \\ delta function \\ sine function \\ Markhov random fields \\ Fourier transform \\ inverse Fourier transform \\ linear TFA
}


laboratory, or in the room next door, etc.). Something in the input is causing the output; we have a situation that demands explanation.

Conversely, bumps or troughs with small coherence mitigate against direct causality. Input energy, at these frequencies, is not reflected in output energy. However, small coberence also needs to be explained. That is, why does this input not elicit an output at these frequencies?

\section{Potential role in patient care}

It is not difficult to imagine the development of a computerised system that makes use of TFA and a brief period of (input) stochastic ventilation (randomly varying rate, then tidal volume, then flow, say) to optimise (output) ventilation or circulation parameters for intensive care patients. It might enable ventilator settings that are specifically tailored to the pathophysiology and anatomy of the particular patient rather than being set using rules of thumb.

\section{Conclusions}

Dr Fujiwara's paper introduces a potentially-important scientific technique to our specialty. Transferfunction analysis holds promise of clarifying why so many of the drugs that we inject or vaporise alter the respiratory, cardiovascular and nervous systems in the way they do.

\section{Analyse de la fonction de transfert en recherche anesthésique}

Nous devons féliciter Fujiwara et coll. de présenter la technique d'analyse de la fonction de transfert (AFT), ou analyse de réponse fréquentielle, dans notre Journal. ${ }^{1}$ L'AFT aide à déduire des informations sur le fonctionnement d'un système en comparant les signaux d'entrée et de sortie. ${ }^{2}$ Elle renseigne mieux sur les processus physiologiques qui y sont dissimulés que ne le fait la simple étude des signaux de sortie. C'est un procédé non effractif, malgré l'utilisation possible de signaux de moniteur effractif. Cette analyse peut fournir une compréhension exceptionnelle de nombreuses situations où les médicaments modifient les interactions complexes des fonctions physiologiques périodiques qui nous intéressent particulièrement (circulation, respiration, nombre de régulateurs neurologiques).

Relativement nouvelle en recherche médicale, ${ }^{3}$ l'AFT n'est nullement "expérimentale». Bien démontrée en ingénierie depuis de nombreuses années, ses subtilités sont connues, ses défauts et ses inconvénients éliminés depuis longtemps. Ses fondements mathématiques sont encore plus anciens (entre autres, Euler, $1707-1783$; Fourier, $1786-1830$ ).

\section{Le signal}

C'est par le signal que sont transmises les informations. ${ }^{4}$ Aux fins de la discussion, nous nous limiterons aux nombreux processus physiologiques qui peuvent être étudiés par la transduction d'un signal physique (son, pression, mouvement, etc.) en un changement de voltage (une variable dépendante en ordonnée) dans le temps (une variable indépendante en abscisse) et transmissible à un ordinateur par digitalisation. Les diagrammes montrent donc, pour des stimuli physiologiques (entrée) qui causent ou influencent des réponses physiologiques (sortie) :

Un stimulus physiologique $\rightarrow$ un transducteur $\rightarrow$ un changement de voltage $\rightarrow$ un analogue de dispositif digital qui prélève rapidement et régulièrement des échantillons de voltage $\rightarrow$ un fichier d'ordinateur contenant un ensemble de nombres.

Chaque nombre, résultat de la digitalisation, est un échantillon du voltage au moment de son enregistrement. Ainsi, chacun renseigne sur l'importance du voltage et le moment où il occupe cette position dans la liste de nombres. Cette liste est un vecteur.

$\mathrm{La}$ réponse physiologique changeante doit subir simultanément la transduction et la digitalisation qui fourniront le vecteur de sortie. L'analyse de la fonction de transfert compare les vecteurs d'entrée et de sortie. La transduction et la digitalisation comportent toutes deux des erreurs d'échantillonnage d'amplitude et de temps. Avec les bons instruments, les erreurs sont minimes, normalement distribuées et aléatoires. Par contre, avec des instruments moins bons, les erreurs sont importantes et/ou comportent des biais systématiques.

Le tracé unidimensionnel du voltage en ordonnée vs le temps en abscisse indique le signal du domainetemps. Ce signal appartient à deux catégories principales : déterministe (une relation mathématique fournit une valeur spécifique de voltage à chaque temps de mesure) et aléatoire ou stochastique (la valeur à un temps donné peut être décrite seulement par une probabilité statistique). Les signaux déterministes sont à leur tour classifiés, sommairement, en périodiques 
(ont un modèle répétitif régulier), ou transitoires (ne l'ont pas). En mathématiques, les signaux périodiques sont des fonctions périodiques formées de la somme des sinus et des cosinus possédant les fréquences et les amplitudes appropriées. L'AFT s'accorde plus facilement aux études des effets d'un système sur des signaux périodiques. Une technique apparentée, la convolution/déconvolution, convient mieux à l'étude des effets d'un système sur des signaux transitoires. Les remarques sur l'utilité de l'AFT en recherche anesthésique s'appliquent également à la convolution/déconvolution.

La théorie de l'AFT suppose que les signaux sont en équilibre dynamique : les signaux périodiques produisent une forme d'onde qui se répète constamment ou les signaux stochastiques changeants présentent des probabilités statistiques constantes. L'étude de l'effet d'un médicament passe donc par la comparaison des vecteurs d'entrée et de sortie, par l'AFT, avant l'administration du médicament et, encore, après qu'un état d'équilibre physiologique est atteint avec le médicament en circulation. Ensuite, on compare les AFT pour déduire l'effet médicamenteux.

L'analyse de la fonction de transfert est de beaucoup simplifiée si le signal d'entrée est un signal blanc connu. Ainsi, Fujuwara se sert pour son étude d'un train d'ondes respiratoires blanches, connues, générées de façon aléatoire. La lumière blanche contient une énergie équivalente à toutes les fréquences. D'où les signaux d'énergie égale à toutes les fréquences sont appelés blancs. En théorie, les signaux blancs renferment toutes les fréquences, de zéro à l'infini. Si l'énergie ne se manifeste qu'à l'intérieur d'un intervalle ou d'une bande de fréquences, le son est dit limité à une bande à l'intérieur de cet intervalle.

\section{Phase}

Nombre d'anesthésiologistes interprètent d'habitude les spectres représentant l'amplitude des potentiels électriques de l'électroencéphalogramme à partir des transformées de Fourier où l'information sur la phase n'est pas retenue. Les spectres de phase sont moins intuitifs. Les signaux périodiques ont un cycle récurrent d'amplitude de voltage à un intervalle de temps régulièrement récurrent ( $=1 /$ fréquence) ; ils sont donc, à tout temps donné, dans une phase spécifique de leur cycle. On peut ainsi en étudier la phase et l'amplitude, et leurs relations. Ces relations, difficiles à discerner dans un graphique du domaine-temps, apparaissent mieux dans un graphique du domaine-fréquence qui illustre à la fois l'amplitude et la phase $v s$ la fréquence. Le graphique de l'AFT est un graphique spécial du domaine-fréquence qui montre le décalage de phase et la mise à l'échelle de l'amplitude de l'entrée vers la sortie pour chaque fréquence.

Les transformations du signal d'entrée d'un système en vue de produire un signal de sortie peuvent prendre du temps. Ce délai décale la phase des composants sinusoïdaux à la sortie créant un déphasage dépendant de la fréquence. En conséquence, on s'attendrait à ce qu'un signal d'entrée commande toujours le signal de sortie sur le graphique de phase de la fonction de transfert. Parfois, c'est la phase de sortie qui semble commander celle de l'entrée. Cela peut se produire de trois façons apparentées : 1) l'entrée commande la sortie, avec le temps, par plus de $180^{\circ} ; 2$ ) l'entrée et la sortie sont inversement reliées (inversion de signal) avec un léger délai effectif ; et 3) il y a une erreur de mesure. Voici quelques exemples simples :

1) LONGS RETARDS DE PHASE. Si l'entrée contrôle la sortie par plus de $180^{\circ}$, un faux délai suit l'application de l'AFT. Sur un cadran d'horloge, avançons de $180^{\circ}$ de 12 à 6 et reculons de $180^{\circ}$ de 12 à 6 . Si un événement déclencheur survient à $12 \mathrm{~h}$ (entrée de degré zéro) et produit une réponse neuf heures plus tard, à $9 \mathrm{~h}$, (une sortie à -90 degrés), l'événement précède, en fait, la réponse de neuf heures. Mais la convention de l'AFT veut que : les cycles de sortie qui présentent des décalages négatifs de phase soient vus comme commandant l'entrée. L'AFT mesure le délai de phase de $12 \mathrm{~h}$ à $9 \mathrm{~h}$ comme moins $90^{\circ}$ et statut que le cycle de sortie précède l'entrée de trois heures. C'est un artéfact des longs retards de phase des systèmes périodiques en état d'équilibre.

Remplissez d'eau un long ballon. D'une main, faites un léger massage cardiaque néonatal à deux doigts, selon diverses fréquences et amplitudes, à l'une des extrémités du ballon (entrée périodique) pendant que l'autre main détecte délicatement le mouvement de l'onde (sortie périodique) transmise à la deuxième extrémité. Il y a un retard évident de l'onde d'entrée à atteindre l'autre extrémité et, à certaines fréquences et amplitudes, l'entrée est «minimale» tandis que la sortie est «maximale» à cause du véritable délai inhérent au système. Le signal de sortie peut donc sembler commander le signal d'entrée.

2) INVERSION DE SIGNAL. Maintenant, figurez-vous un jeu de bascule activé à une extrémité (entrée) pour produire un balancement sinusoïdal. Le mouvement vertical est transmis à chaque extrémité. Le signal de sortie est en phase de $180^{\circ}$ avec le signal d'entrée, mais comme le système produit une inversion totale et instantanée du signal, il n'y a pas de véritable retard. La transduction du mouvement vertical des points de repère d'un système à deux dispositifs engrenés ( $\mathrm{I}^{\text {er }}$ 
dispositif : entrée : repère à $12 \mathrm{~h} ; 2^{\mathrm{e}}$ dispositif : sortie : repère à $9 \mathrm{~h}$ ) peut créer une inversion instantanée partielle du signal, de sorte que le signal de sortie semble précéder le signal d'entrée.

3) L'ERREUR DE MESURE. Aux fréquences où il n'y a qu'un très faible transfert d'énergie (faible $|\mathrm{H}(\mathrm{q})|$, l'amplitude dans l'AFT), les informations de phase amplifient l'erreur d'échantillonnage par une sorte de parallaxe mathématique. Une faible valeur de $|\mathrm{H}(\mathrm{q})|$ provoque une information de phase vide de sens, c'est pourquoi l'amplitude et la phase doivent toujours être considérées ensemble.

Finalement, pour compliquer les choses, des combinaisons de ces trois mécanismes peuvent survenir. D'où l'utilité d'en connaître le plus possible sur le fonctionnement du système afin d'interpréter correctement les changements de phases.

\section{Le système}

Du point de vue mathématique, un système change un, ou plusieurs, signal d'entrée en un, ou plusieurs, signal de sortie. De nombreux processus physiologiques, dans leurs fonctions habituelles, au moment où les organismes «vivent» le plus intensément leur vie, montrent des réponses linéaires aux influences qui les modifient ; c'est-à-dire que la réponse est proportionnelle à l'influence. Ce qui permet d'utiliser les modèles mathématiques selon des méthodes linéaires comme l'AFT et la convolution/déconvolution. Les systèmes linéaires démontrent les propriétés de l'ajustement (la multiplication du voltage par un facteur d'échelle est valide) et de la superposition ou de l'additivité (la réponse à la somme de deux signaux d'entrée est la somme des réponses à chaque signal).

\section{Le processus}

En bref, on compare le signal de sortie au signal d'entrée en utilisant d'abord une transformée rapide de Fourier, une modalité à base de nombres complexes, pour transformer les vecteurs d'entrée et de sortie en fonctions de domaine-fréquence qui conservent leurs informations d'amplitude et de phase. La fonction qui transforme le signal d'entrée en signal de sortie (la fonction du système) est alors estimée en divisant la fonction de sortie, évaluée en nombres complexes, par la fonction d'entrée, évaluée en nombres complexes.

La validité statistique du processus est ensuite vérifiée par une version à nombre complexes de la régression linéaire, appelée fonction de cohérence, laquelle rapporte une valeur entre zéro et un. Il y a faible cohérence quand 1) un signal d'entrée ou de sortie est contaminé par le bruit, ou 2) le signal de sortie n'a pas de lien de cause à effet avec le signal d'entrée, ou 3) le système n'est pas linéaire. Une grande cohérence signifie une forte probabilité que le signal de sortie soit en lien de cause à effet avec le signal d'entrée et que le système soit linéaire.

Les graphiques sont ensuite examinés et des déductions faites sur le fonctionnement du système. Plus on en sait sur la structure et le fonctionnement du système physiologique, plus il est possible d'interpréter correctement l'AFT.

\section{Equivalences terminologiques}

Terme

Signal périodique

Fréquence du signal périodique

Amplitude du signal périodique

Phase du signal périodique

Signal d'entrée transitoire

Signal de sortie transitoire

Signal deterministe

Signal stochastique

Temps nécessaire à la

transformation au

domaine-fréquence

Fréquence nécessaire à la

transformation au

domaine-temps

AFT

\section{Physiologie \\ pulsation \\ hauteur musicale}

intensité du son (puissance)

délai entre les noeuds sinusal et AV

bolus

ronchus

pulsation générée par un

stimulateur cardiaque

suite de pulsations dans la fibrillation

cochlée de l'oreille interne

activité neuronale motrice des jambes d'un cycliste

lumière du soleil au travers de l'eau (voir texte)

\section{Ingénierie}

ondes (tous médias)

courant alternatif de «60 cycles»

voltage du CA (non la puissance)

décalage entre le son et son écho

explosion sismique provoquée

effondrement d'un pont

générateur d'onde sinusoïdale

auriculaire murmure d'un ruisseau

émetteur FM

récepteur FM

essai avec équipement audio

\author{
Mathématiques \\ onde sinusoïdale \\ rotation angulaire de la \\ fonction sinus \\ spectre de puissance \\ angle de phase de la fonc- \\ tion sinus \\ fonction delta \\ fonction delta \\ fonction sinus \\ champs aléatoires de \\ Markhov \\ transformée de Fourier
}

transformée inverse de Fourier

AFT linéaire 


\section{Le rôle de l'AFT en recherche anesthésique}

La plupart de nos connaissances de la physiologie et de la pharmacologie proviennent d'expériences sur de jeunes quadrupèdes en santé, anesthésiés aux barbituriques, placés en décubitus dorsal, dans la position la moins naturelle pour eux. Par ailleurs, nos patients sont des bipèdes âgés, malades, placés en position normale. Historiquement, on a extrapolé aux humains les découvertes faites chez les animaux avec assez de succès. Mais peut-être peut-on faire mieux. L'AFT n'est pas un procédé effractif. Un modèle expérimental, semblable à celui de Fujiwara, peut servir à l'étude de nombreux médicaments qui ont des effets sur la circulation chez les humains. Il peut aussi faire mieux comprendre leurs effets physiologiques, en particulier dans certaines populations (nouveau-nés, personnes âgées, patients atteints d'athérosclérose par exemple) qui pourraient réagir différemment des jeunes animaux adultes en laboratoire.

Il serait particulièrement intéressant d'appliquer cette technique aux patients qui souffrent de fibrillation auriculaire et qui nous fournissent un signal connu, survenant naturellement et au hasard sous forme de variabilité de la fréquence cardiaque. Aussi, un signal d'entrée stochastique, plutôt qu'un signal régulier et périodique utilisé généralement, est facile à programmer pour l'obtention de potentiels évoqués visuels, auditifs et somesthésiques. L'AFT peut sûrement clarifier les eaux troubles des interactions médicamenteuses avec les potentiels évoqués.

\section{Mise en garde}

Rien n'est vraiment linéaire dans le monde naturel. Bien que de nombreux phénomènes s'en approchent suffisamment pour être étudiés selon des méthodes linéaires, certains ne sont pas linéaires. Nous devons toujours considérer les résultats avec prudence et vérifier nos hypothèses le mieux possible. Rappelons aux chercheurs que l'AFT doit être mise à l'essai et vérifiée à partir du déjà connu. Ce n'est pas une boîte magique qui donne des réponses sans qu'on ait à réfléchir.

Nous devons scruter le graphique de la fonction $|\mathrm{H}(\mathrm{q})|$ et donner une explication physique à ses creux et bosses. Ainsi, nous pouvons voir l'eau de mer bleue/verte quand le soleil (lumière blanche) y pénètre, nous faisons l' «analyse de la fonction de transfert» dans notre cortex visuel. Mais pour dire pourquoi l'eau est bleue/verte, nous devons alors comprendre le comportement des photons dans cette eau : un pas difficile à franchir. L'AFT permet habituellement de mettre l'accent avec justesse sur les questions à venir plutôt que de fournir des réponses mécanistes.
Les points maximal et minimal de $|\mathrm{H}(\mathrm{q})|$, peu importe où se situe la plus grande cohérence, nous indiquent que c'est une fréquence que nous devons expliquer. Pour trouver l'explication, nous observons les processus physiologiques qui présentent ces fréquences (ou un bruit contaminant d'autres instruments dans le laboratoire, ou dans la pièce à côté, etc.). Quelque chose dans le signal d'entrée produit le signal de sortie ; nous devons expliquer cette situation.

Inversement, les points maximal et minimal de faible cohérence militent contre une causalité directe. L'énergie initiale, pour ces fréquences, n'est pas retrouvée dans l'énergie finale. Cependant, une faible cohérence nécessite également des explications. C'est-àdire, pourquoi, selon ces fréquences, l'énergie de départ ne déclenche-t-elle pas de signal de sortie?

\section{Applications cliniques possibles}

On imagine facilement le développement d'un système informatique qui utilise l'AFT et une brève période de ventilation (signal d'entrée) stochastique (par exemple, une fréquence, un volume courant, un débit de variabilité aléatoire) afin d'optimaliser (signal de sortie) les paramètres de ventilation ou de circulation chez les patients requérant des soins intensifs. On pourrait done régler la ventilation en l'adaptant précisément aux besoins physiopathologiques et anatomiques d'un patient en particulier plutôt que de répondre à des règles générales.

\section{Conclusion}

L'article du docteur Fujiwara présente une technique scientifique potentiellement importante. L'analyse de la fonction de transfert est prometteuse, car elle pourrait expliquer pourquoi tant de médicaments injectés ou vaporisés modifient les systèmes respiratoire, cardiovasculaire et nerveux comme ils le font.

\section{References}

1 FujiwaraY. Transfer function analysis of the circulation in patients undergoing sevoflurane anesthesia. Can J Anesth 1999; 46: 820-19.

2 Parker JA. Image Reconstruction in Radiology. Boca Raton Fla USA: CRC Press Inc., 1990.

3 Berger RD, Saul JP, Coben RJ. Transfer function analysis of autonomic regulation. I. Canine atrial rate response. Am J Physiol (Heart Circ Physiol 25)1989; 256: H142-52.

4 Cohen A. Biomedical Signal Processing, Vol I. Boca Raton Fla USA: CRC Press Inc., 1986. 